\title{
Vacuum-Deposited Multication Tin-Lead Perovskite Solar Cells
}

Ana M. Igual-Muñoz ${ }^{1}$, Aroa Castillo ${ }^{1}$, Chris Dreessen ${ }^{1}$, Pablo P. Boix*2, and Henk J. Bolink*1.

${ }^{1}$ Instituto de Ciencia Molecular, Universidad de Valencia, C/J. Beltran 2, 46980 Paterna, Spain.

${ }^{2}$ Institut de Ciència dels Materials, Universitat de València, C/ J. Beltran 2, 46980 Paterna, Spain.

*E-mail: Pablo.P.Boix@uv.es; $\underline{\text { henk.bolink@uv.es }}$

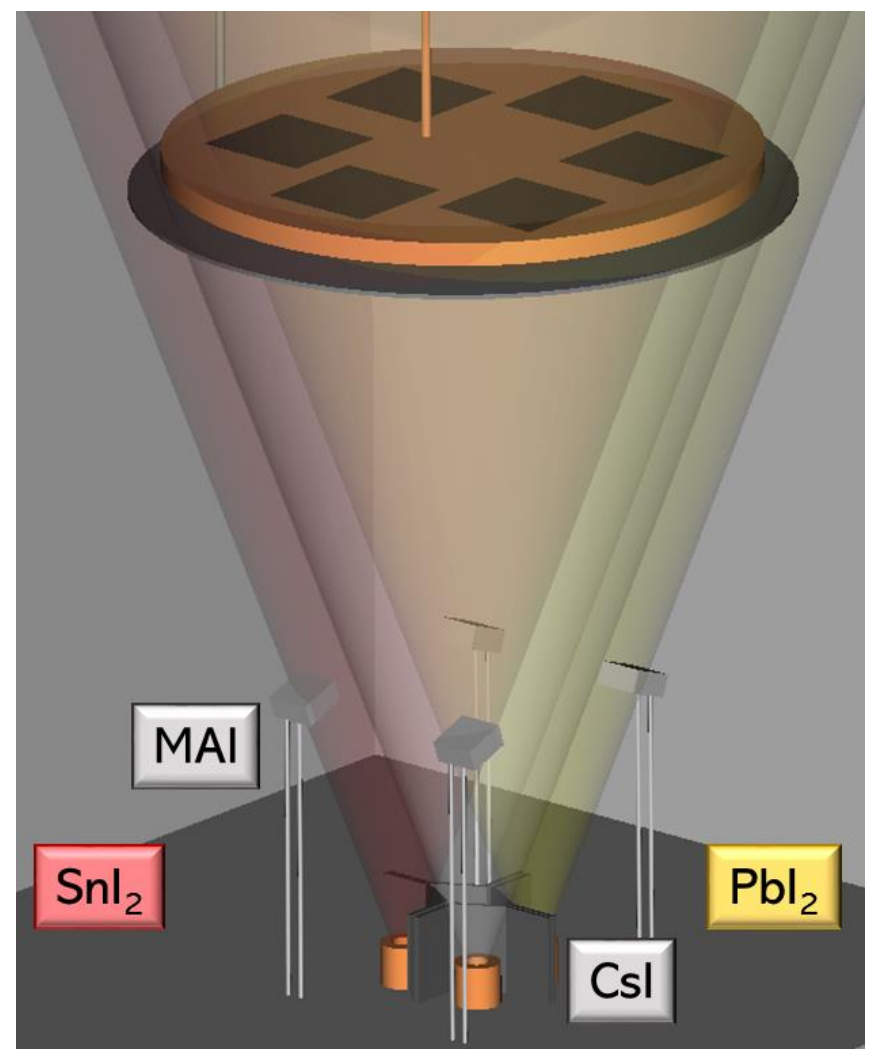

Figure S1. Scheme of the evaporator machine. 
a.

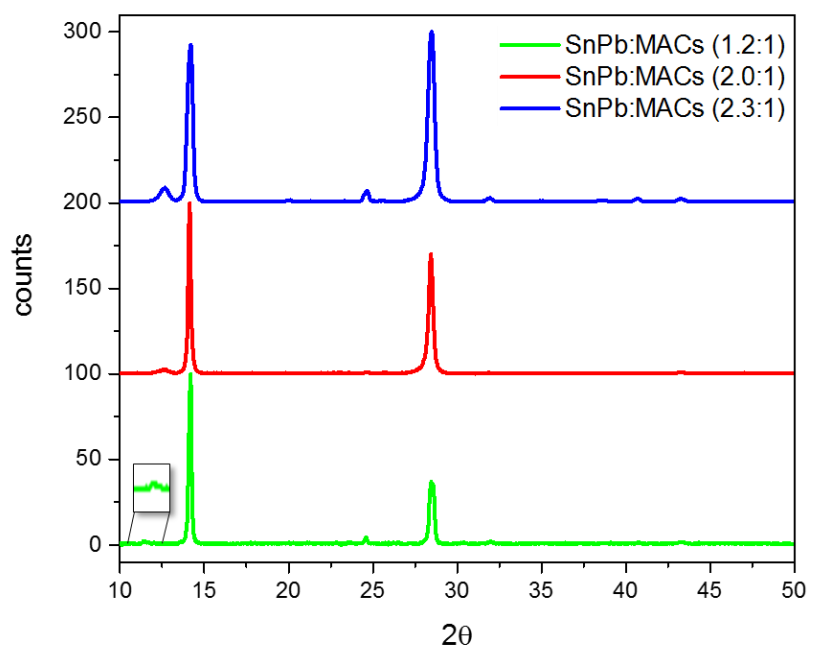

b.

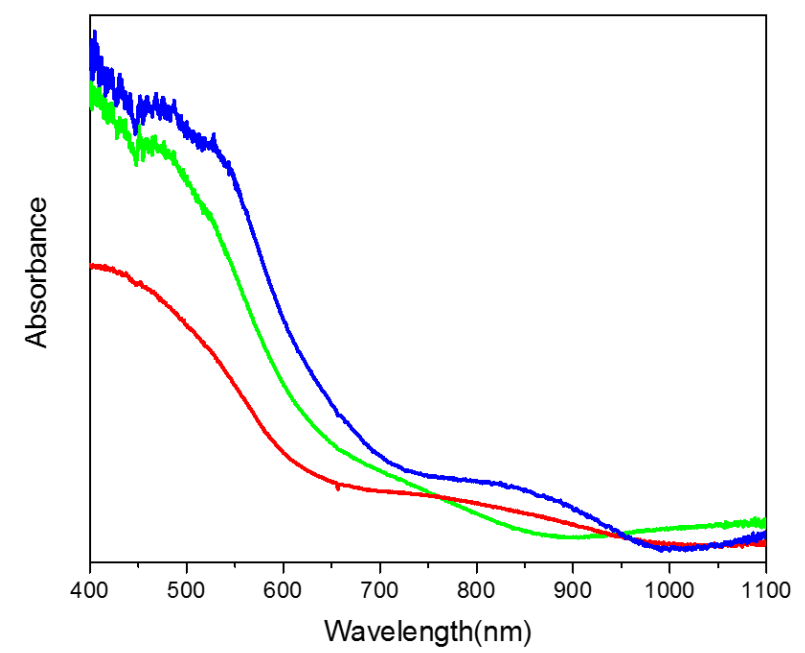

Fig. S2. a) XRD diagrams and b) absorbance of $\mathrm{MA}_{0.9} \mathrm{CS}_{0.1} \mathrm{Sn}_{0.25} \mathrm{~Pb}_{\left.0.75\right|_{3}}$ films with different $\mathrm{SnPb}: \mathrm{MACs}$ ratios.
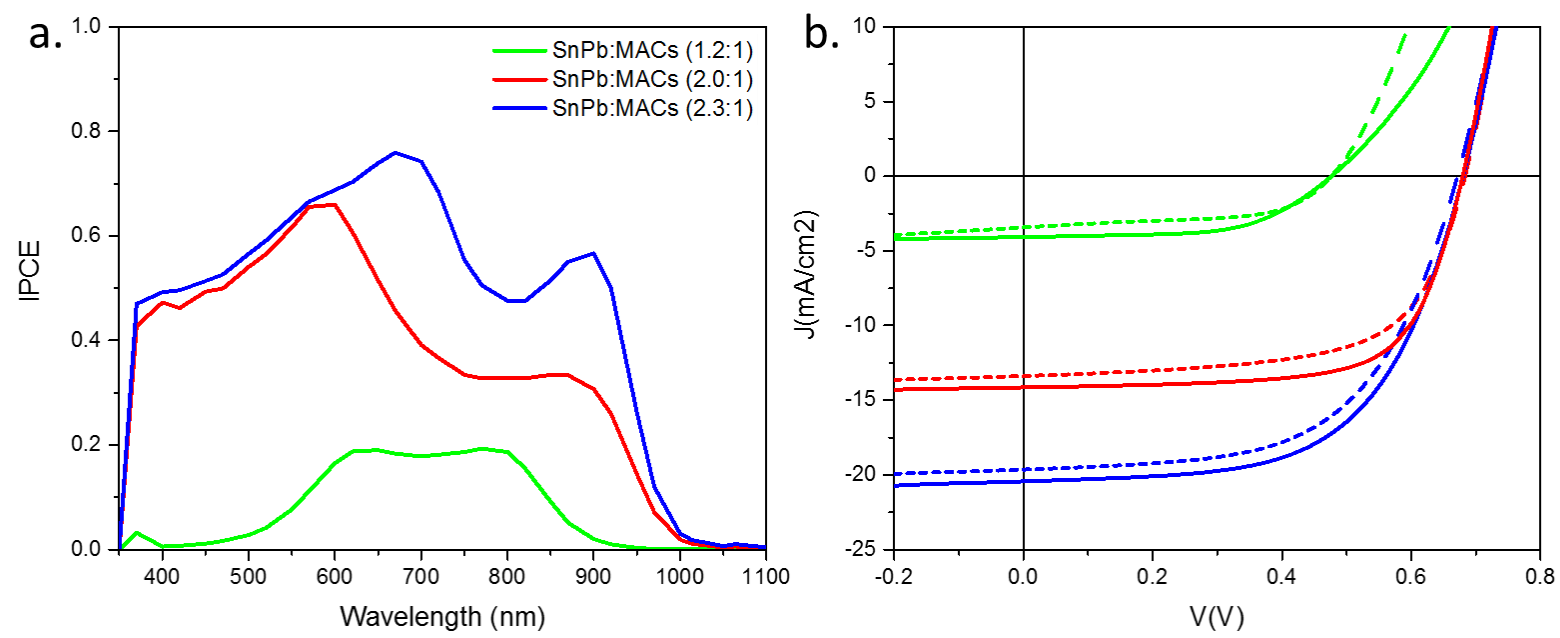

Fig. S3. (a) External quantum efficiency (EQE) and (b) JV measurement under light conditions for the solar cells made with different SnPb to MACs ratios. Dashed lines represent backward measurements and solid lines represent forward measurements (from negative to positive potential).

Table S1. Photovoltaic parameters under AM1.5G illumination measured for the solar cells represented in Fig. S2.

\begin{tabular}{ccccc}
\hline Ratio (SnPb:MACs) & $\mathrm{FF}(\%)$ & $\mathrm{J}_{\mathrm{sc}}\left(\mathrm{mA} / \mathrm{cm}^{2}\right)$ & $\mathrm{V}_{\mathrm{oc}}(\mathrm{mV})$ & PCE (\%) \\
\hline $1.2: 1$ & 57 & 3.7 & 477 & 1.0 \\
$2.0: 1$ & 66 & 13.7 & 681 & 6.1
\end{tabular}


a.

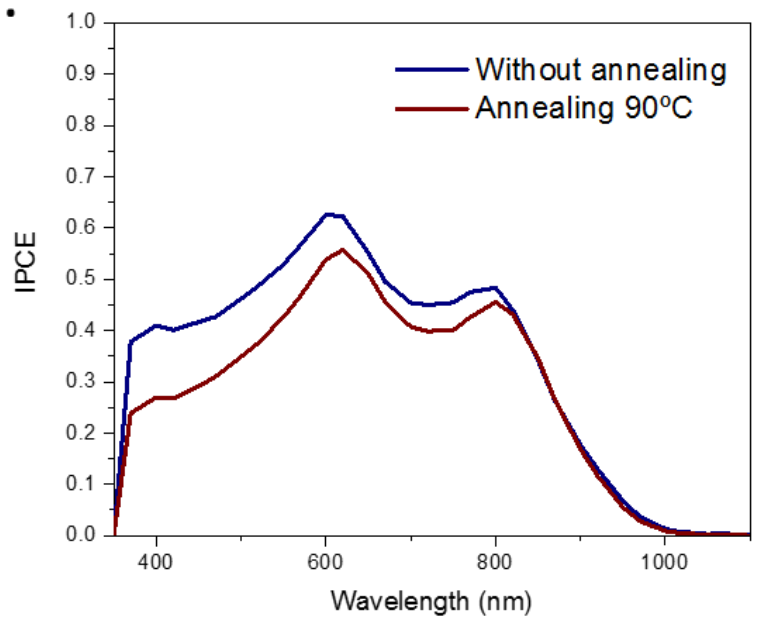

b.

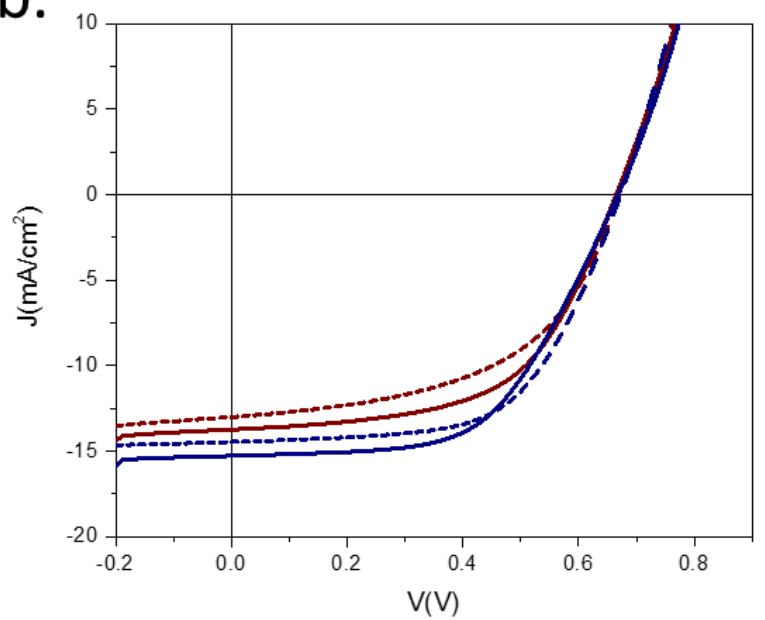

Fig. S4. J-V characteristics measured in light conditions for cells prepared with and without thermal post-treatment of 90 으 for 5 minutes.

a.

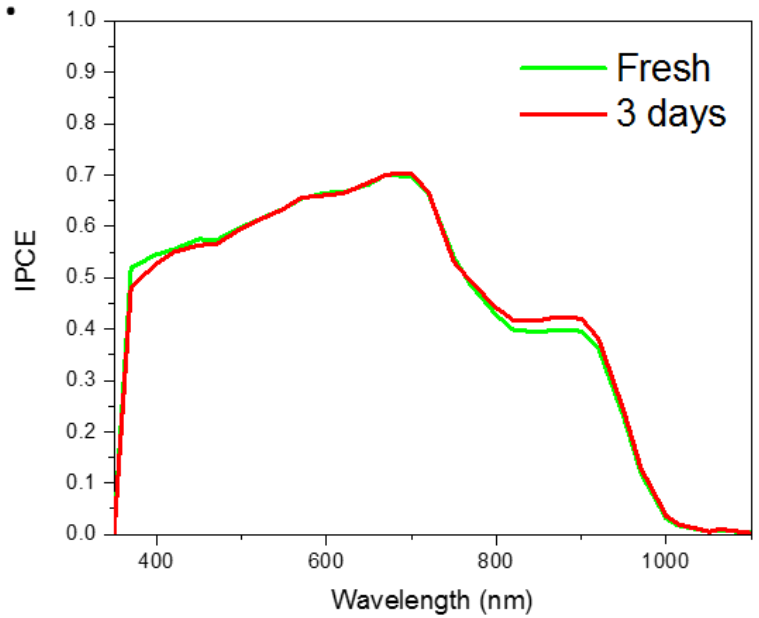

b.

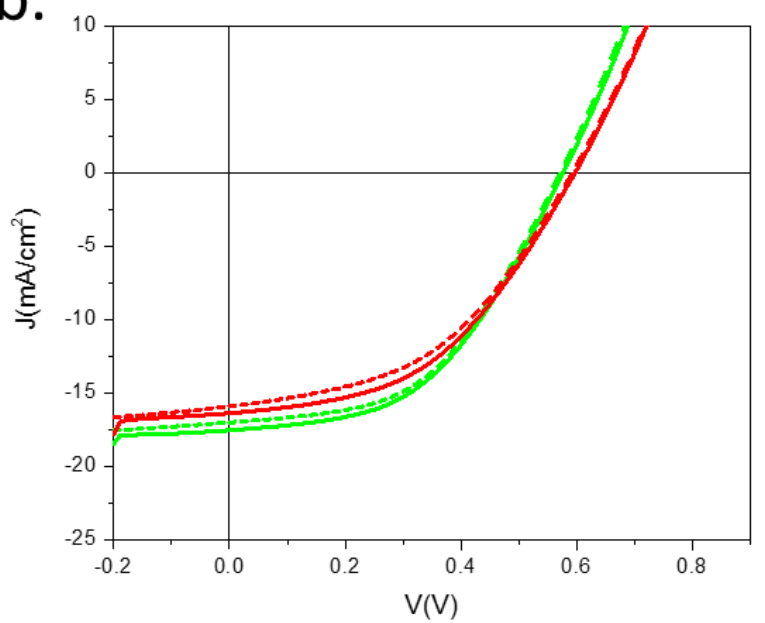

Fig. S5. J-V characteristics measured in light conditions for cells as prepared and after three days of storage in a glovebox containing $\mathbf{N}_{2}$. 


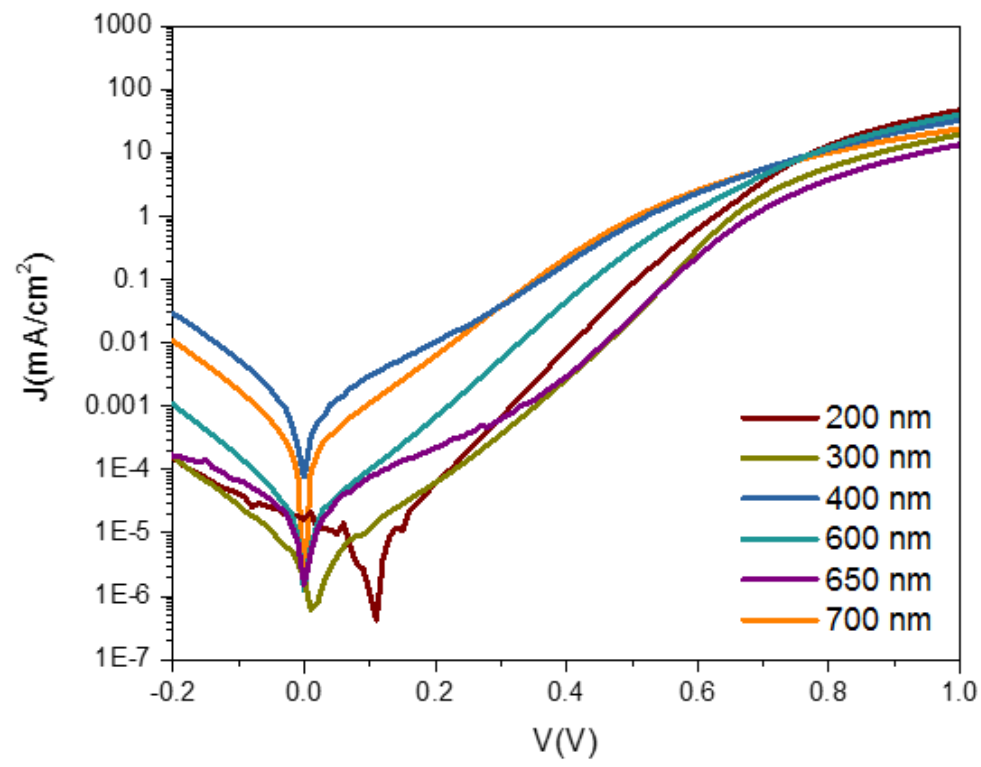

Fig. S6. J-V characteristics measured in dark conditions for the representative devices with different thicknesses.

a.
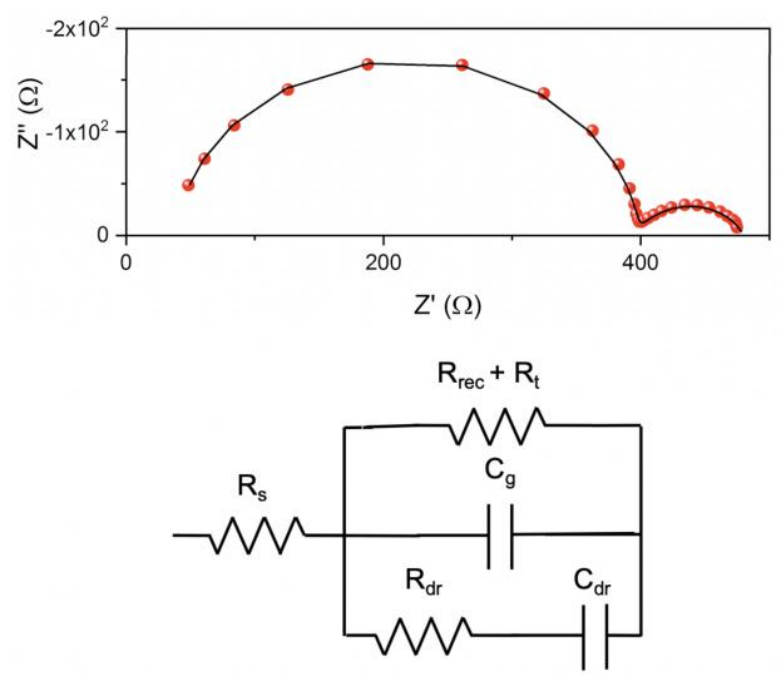

b.

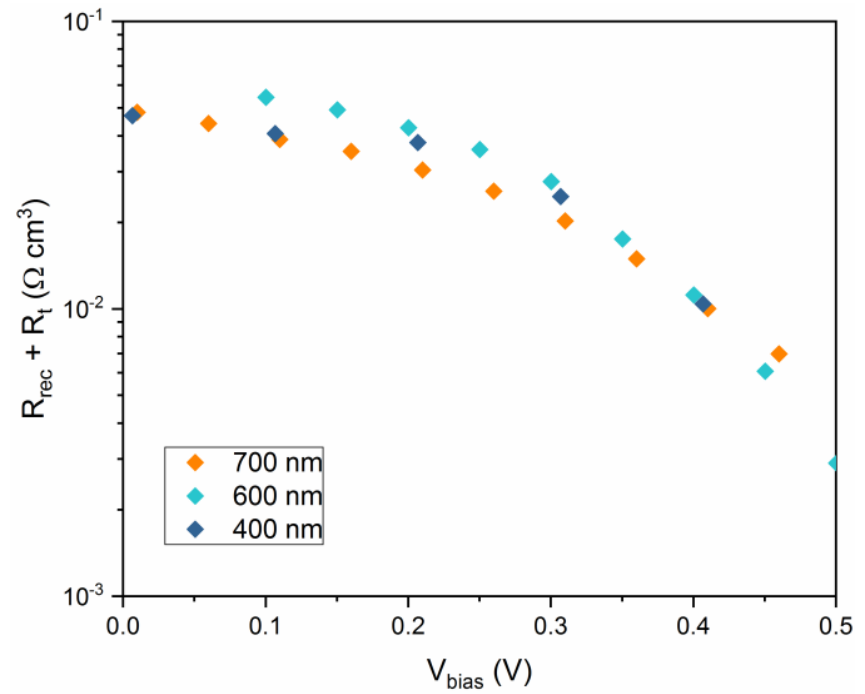

Fig. S7. (a) Representative Nyquist plot obtained for the $600 \mathrm{~nm}$ sample under 0.5 sun illumination. The dots correspond to the experimental data and the solid line is the fitting using the equivalent circuit in (b). (c) Volumetric normalization of $R_{r e c}+R_{t}$. 


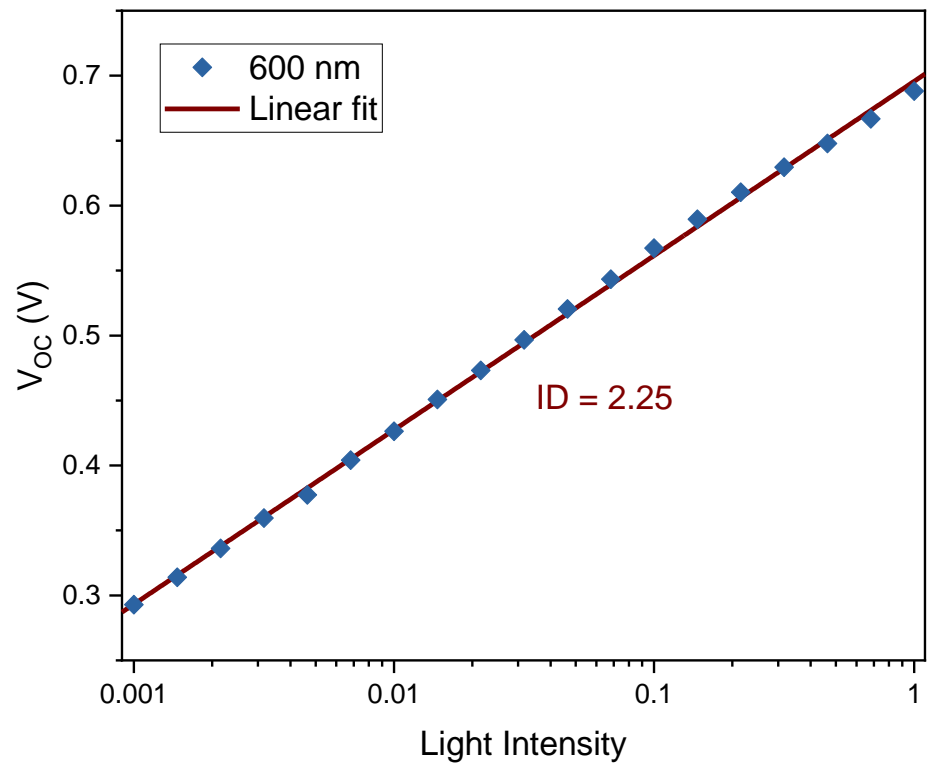

Figure S8. Voc vs the logarithm of light intensity for the determination of the ideality factor. Note that a light intensity of 1 corresponds to more than one sun illumination. 